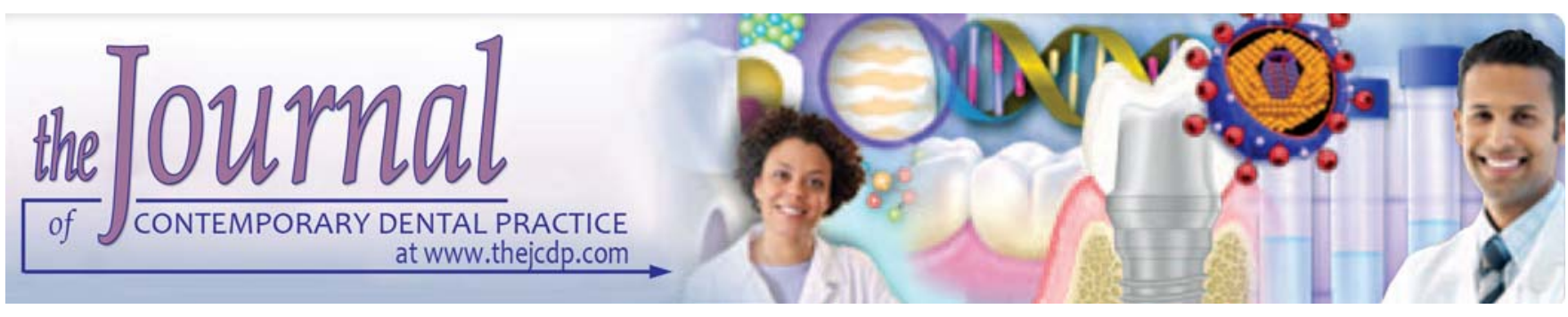

\title{
Effects of Citric Acid and EDTA on Periodontally Involved Root Surfaces: A SEM Study
}

Sunkara Shree Ramalinga Prasad, Chitturi Radharani, Siddhartha Varma, SV Kiran Kumar

Soumya Sinha, Bijle Mohammed Nadeem Ahmed

\section{ABSTRACT}

Aim: Root debridement by scaling and root planing may not completely remove contaminated cementum particularly in more apical areas. The smear layer formed during root planing inhibits cell migration and attachment leading to impaired marginal periodontal healing. The present study was done to compare the morphological effects of root surface demineralization using citric acid and EDTA as root-conditioning agents.

Materials and methods: Thirty fragments of human dental roots previously exposed to periodontal disease were scaled and randomly divided into the following groups of treatment: Group I: Hand instrumentation and conditioning with normal saline for 5 minutes as control; group II: Hand instrumentation and conditioning with citric acid ( $\mathrm{pH} \mathrm{1)}$ for 5 minutes and group III: Hand instrumentation and conditioning with EDTA ( $\mathrm{pH}-7.4)$ for 5 minutes. Scanning electron microscopy was used to check for the presence of residual smear layer and for measuring the number and area of exposed dentin tubules.

Results: The mean efficacy of smear layer removal was compared between groups I and II, groups I and III and groups II and III, it was found to be statistically significant at $5 \%$. When the mean diameter of the dentinal tubules and mean total surface area occupied by the dentinal tubule orifices was compared between groups II and III it was found to be statistically significant at $1 \%$ level of significance.

Conclusion: Citric acid causes greater degree of morphological alterations than EDTA and is considered to be a better rootconditioning agent. However, the use of EDTA cannot be ruled out.

Clinical significance: This study supports the hypothesis that demineralizing agents can be used as an adjunct to the periodontal treatment aiming at restoring the biocompatibility of roots and helps in choosing an appropriate agent for root conditioning.

Keywords: Root planning, Smear layer, R oot conditioning.

How to cite this article: Prasad SSR, R adharani C, Varma S, Kumar SVK, S inha S, Bijle MNA. E ffects of Citric Acid and EDTA on Periodontally Involved Root Surfaces: A SEM study. J Contemp Dent Pract 2012;13(4):446-451.

Source of support: Nil

Conflict of interest: None declared

\section{INTRODUCTION}

Periodontitis-affected cementum presents loss of collagen fiber insertion, may harbor bacterial cells and may be contaminated by endotoxins which may suppress fibroblast migration and proliferation on cementum. R oot debridement by scaling and root planing may not completely remove contaminated cementum particularly in more apical areas and the smear layer formed during root planing inhibits cell migration and attachment leading to impaired marginal periodontal healing. ${ }^{1}$

There is considerable interest in the use of chemical agents to assist in root preparation. A gents have been proposed to accomplish the following:

- Eliminate cytotoxic material from affected root surfaces

- Clean the exposed dentin surfaces

- Decalcify planed root surfaces exposing dentine or cementum matrix collagen and thus facilitate attachment between the root surface and the healing connective tissue. $^{7}$

In the present study, an attempt is made to compare the morphological effects of root surface demineralization using citric acid and EDTA as root-conditioning agents.

Objective of the study:

- To test efficacy of smear layer removal.

- Appreciation of collagen fiber-like structures within intertubular dentin.

- Evaluate number of dentinal tubule orifices.

- Estimate diameter of individual dentinal tubules.

- Evaluate total surface area occupied by the dentinal tubules.

\section{MATERIALS AND METHODS}

Fifteen extracted single-rooted human teeth of 12 subjects with advanced periodontitis were collected from the 
Effects of Citric Acid and EDTA on Periodontally Involved R oot Surfaces: A SEM study

Department of Oral Surgery, SDM College of Dental Sciences, Dharwad.

\section{Criteria for Selection of the Teeth}

- No history of root planing, scaling and prophylaxis in the previous 6 months

- No history of acute pain or swelling necessitating their removal

- Proximal attachment loss of $5 \mathrm{~mm}$ or more

- Bleeding on gentle probing

- A bscence of caries on the proximal surface selected for the study

- No history of use of antibiotics during the previous 6 months

- No history of any systemic diseases.

The extracted teeth were placed in sterile vials containing normal saline solutions which were processed without delay.

\section{Preparation of Teeth}

The teeth were cleaned of blood and saliva with a soft bristled brush and distilled water. A dissecting microscope was used to determine the level of connective tissue attachment and that was marked with a small dental bur. The reference groove was then accentuated by deepening the groove with a diamond disk in high speed hand piece under continuous water coolant. The proximal surfaces of each tooth were vigorously root planed with Gracey N 0.1 and 2 hand curette which was resharpened after each block in an attempt to remove all cementum and achieve a smooth, hard, glass-like surface.

The instrumented surfaces were then re-examined to ensure removal of all calculus. U sing a high speed handpiece with copious water coolant the crowns were resected at the cementoenamel junction (CEJ). To provide an experimental surface, each tooth was sectioned longitudinally as 2 parts, and the pul pal side was flattened with a straight bur followed by a small inverted cone to make a horizontal groove on the pulpal surface for identification purpose. Lastly, these specimen were harvested by a horizontal cut in the coronal $1 / 3$ rd of each tooth half, to yield dentin slabs about $4 \times 4$ $\mathrm{mm}$ in size. This yielded a total of 40 dentin slabs. Then each tooth section was then scrubbed with a soft brush and distilled water to remove grinding debris.

\section{Preparation of Acid Solutions}

A citric acid solution was prepared by slowly adding $65 \mathrm{gm}$ of citric acid crystals into $100 \mathrm{ml}$ of distilled water and stirred constantly. This gave a pH 1 solution, when checked with a $\mathrm{pH}$ meter.
EDTA solution was prepared by slowly adding $24 \mathrm{gm}$ of disodium salt of EDTA to $100 \mathrm{ml}$ of distilled water and stirred constantly. This gave a pH of 7.4 when checked with a pH meter. Both the solutions were prepared immediately before the experimental treatment.

Out of the 30 dentine slabs, 10 sections were randomly assigned to the following three treatment groups

- Group I: Hand instrumentation and conditioning with normal saline for 5 minutes as control

- Group II: Hand instrumentation and conditioning with citric acid (pH 1) for 5 minutes

- Group III: Hand instrumentation and conditioning with EDTA (pH - 7.4) for 5 minutes.

\section{List of Armamentarium}

- Soft bristle brush

- Dissecting microscope

- Sterile vials

- Diamond-cutting disks and cone bur

- Gracey's No I and II hand curette

- Tweezer and cotton pellets

- pH meter

- Electron microscope.

\section{Chemical Treatment of Root Surfaces}

The acid solutions were applied for 5 minutes to the experimental root surfaces with cotton pellets moistened with the agent, and the cotton pellets were changed 2 times per minute. These solutions were applied with light pressure to allow the agent to wet the surface without burnishing the agent into the root surface. A dditional tooth sections served as controls and treated with cotton pellets moistened with normal saline solution. A fter conditioning, specimens were rinsed for 2 minutes with distilled water.

\section{Preparation for SEM Study}

A fter treatment of the root surfaces, samples were fixed in $2.5 \%$ gluteraldehyde in phosphate buffer $(\mathrm{pH} 7.3)$ for 24 hours at $40^{\circ} \mathrm{C}$, washed three times for 10 minutes each in phosphate buffer, post fixed in phosphate-buffered 1.5\% osmium tetroxide for 2 hours and washed three more times in phosphate buffer.

The specimens were then dehydrated in a graded series of aqueous ethanol solutions 50, 70, 85, 95 and $100 \%$ ethanol for 10 minutes each. After 2 more 10 minutes washing in absolute ethanol, the samples were dried overnight in a dessicator jar containing silica gel. They were mounted on SEM stubs with silver paint and sputter coated with gold. The mounted slabs were evaluated using a J eol 100 CX SEM . 


\section{SEM Examination}

The presence of smear layer, exposure of the collagen fiberlike structures, the number of dentinal tubules, diameter of the tubules and the average total surface area occupied by the tubules were measured at a magnification of $\times 3,000$ with zero tilt angle.

The efficacy of smear layer removal by using different solutions was estimated by the following scores:

- Score 0: N o patches of smear layer present.

- Score 1: Small patches of smear layer present.

- Score 2: D efinite patches of smear layer present.

- Score 3: Considerable amount of smear layer present.

- Score 4: Completely covered by smear layer.

The appreciations of collagen fiber-like structures within the intertubular area were estimated by using the following scores:

- Score 1: Poor (cannot be appreciated)

- Score 2: M oderate

- Score 3: Good

- Score 4: Excellent.

The diameter of the dentinal tubule orifices, total surface area occupied by the dentinal tubule orifices were measured directly from the screen of the SEM with caliberated scal es. A single investigator performed all morphometric measurements. Total of $100 \times 100 \mathrm{~m}^{2}$ area was measured from each specimen.

\section{RESULTS}

The study consisted of 30 specimens, out of which, 10 specimens were randomly assigned to three treatment groups: Group I-saline, group II-citric acid and group III-EDTA. After the treatment the specimens were immediately prepared for the SEM examination. A single investigator performed all the morphometric analysis.

The mean values obtained for various parameters in the different groups were subjected to statistical analysis by applying Students t-test.

\section{Efficacy of Smear Layer Removal}

When the mean efficacy of smear layer removal was compared between groups I and II, groups I and III and groups II and III, it was found to be statistically significant at $5 \%$ level of significance $(p<0.05)$ as shown in Table 1 (Figs 1 and 2).

\section{Appreciation of Collagen Fiber-like Structures}

When the mean appreciation of collagen fiber-like structures was compared between groups I and II, groups I and III and groups II and III it was found to be satistically significant at $5 \%$ level of significance as shown in Table 2.

\section{Number of Dentinal Tubule Orifices}

When the mean number of dentinal tubule orifices was compared between groups II and III it was found to be statistically not significant as shown in Table 3 .

\begin{tabular}{llllll} 
& \multicolumn{4}{l}{ Table 1: Mean efficacy of smear layer removal: Comparison between different groups } \\
\hline Variables & Mean & SD & t-value & p-value & Significance \\
\hline Saline & 4.00 & 0.000 & 24.2222 & $<0.05$ & $\mathrm{~S}$ \\
Citric acid & 0.3 & 0.4831 & 21.00 & $<0.05$ & $\mathrm{~S}$ \\
\hline Saline & 4.00 & 0.000 & & & \\
EDTA & 1.2 & 0.4217 & -4.4388 & $<.05$ & $\mathrm{~S}$ \\
\hline Citric acid & 0.3 & 0.4831 & & \\
EDTA & 1.2 & 0.4217 & & \\
\hline
\end{tabular}

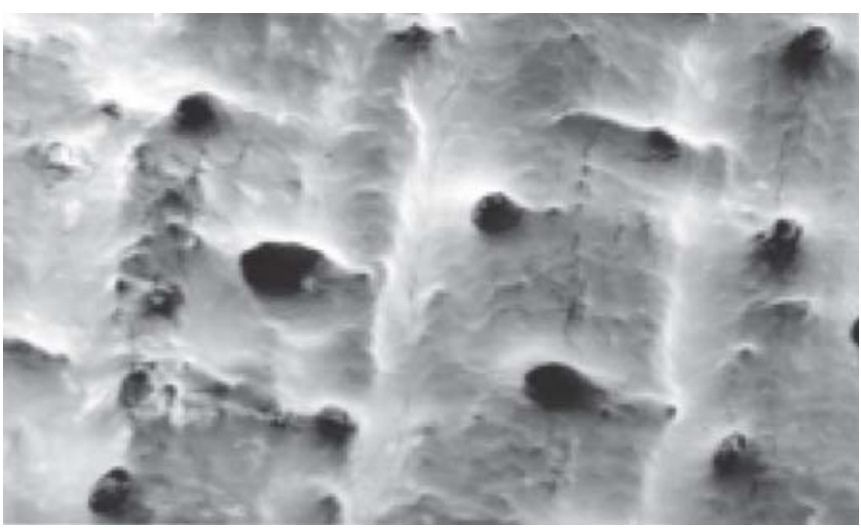

Fig. 1: Group II: Hand instrumented and citric acid-treated surface. It had smooth to undulating surface texture. Round to oval dentinal tubules are evident and devoid of smear layer

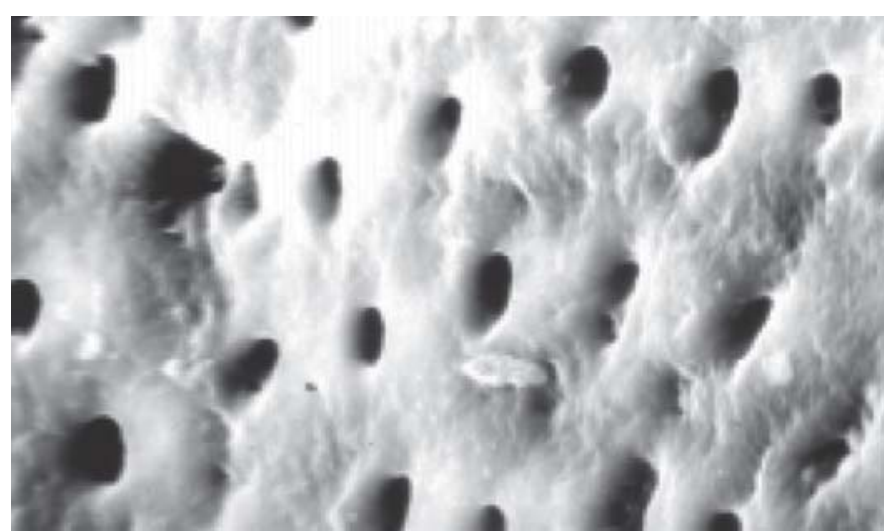

Fig. 2: Group III: Hand instrumented and EDTA treated surface. It had smooth to undulating surface texture. Open dentinal tubules can be appreciated 
Effects of Citric Acid and EDTA on Periodontally Involved R oot Surfaces: A SEM study

\begin{tabular}{lccccc} 
& \multicolumn{2}{c}{ Table 2: Mean appreciation of collagen-like structures: Comparison between different groups } \\
\hline Variables & Mean & SD & t-value & p-value & Significance \\
\hline Saline & 1.00 & 0.000 & -8.5732 & $<0.05$ & $\mathrm{~S}$ \\
Citric acid & 2.24 & 0.5164 & & & $\mathrm{~S}$ \\
\hline Saline & 1.00 & 0.000 & -2.4495 & & \\
EDTA & 1.4 & 0.5164 & & & \\
\hline Citric acid & 2.4 & 0.5164 & 4.3301 & & $\mathrm{~S}$ \\
EDTA & 1.4 & 0.5164 & & & \\
\hline
\end{tabular}

S: Significant

Table 3: Mean comparison of dentinal tubule orifices between different treatment groups

\begin{tabular}{|c|c|c|c|c|c|c|}
\hline Variable & Material & Mean & SD & t-value & $p$-value & Significance \\
\hline Tubule orifice & $\begin{array}{l}\text { Citric acid } \\
\text { EDTA }\end{array}$ & $\begin{array}{l}27.40 \\
25.20\end{array}$ & $\begin{array}{l}7.0110 \\
8.8544\end{array}$ & 0.6159 & $>0.01$ & NS \\
\hline
\end{tabular}

NS: Nonsignificant

\section{Diameter of the Dentinal Tubules}

When the mean diameter of the dentinal tubules was compared between groups II and III it was found to be statistically significant at $1 \%$ level of significance as shown in Table 4.

\section{Total Surface Area Occupied by the Dentinal Tubule Orifices}

When the mean total surface area occupied by the dentinal tubule orifices was compared between groups II and III it was found to be statistically significant at $1 \%$ level of significance as shown in Table 5. Extensive surface cracking was a frequent finding among all the different groups.

\section{DISCUSSION}

The surface appearance of the root-planed specimens in this study resemble those in previous investigations and thus may represent a smear layer. ${ }^{2,3,7}$ Smear layer is formed irrespective of the treatment modality used, ${ }^{3}$ i.e. ultrasonic, curette or diamond bur. In this study, both the acids (citric acid and EDTA ) used were effective in removing the smear layer than the saline-treated group. O ut of these, citric acid had better efficacy in smear removal than EDTA.

The agents used in this study either diminished or completely removed the smear layer and exposed the dentinal tubule. This study is in agreement with the study done by Hanes PJ et al ${ }^{4}$ who had observed some amount of surface debris on specimens treated with different acids.

The method of application has varied among the clinicians, i.e. placement of the agent either passively or burnishing. In the present study, application with light pressure is preferred over burnishing technique as the later may itself form smear layer which may partially or completely obliterate the dentinal tubule openings. Furthermore, both the techniques were found to be equally effective in removing the smear layer in a study done by Rosa wen $\mathrm{C}$ et al. ${ }^{5}$

The results of this study indicate that citric acid causes greater degree of collagen fiber exposure than EDTA. Counting of the dentinal tubule orifices in saline group was not possible as the root surface was covered by smear layer. Hence, comparison was made between the acids used. Comparing the mean number of dentinal tubule orifices exposed there was no statistically significant difference between both the acid groups. This indicated that both the acids were effective in exposing the dentinal tubules almost to the same extent.

Intergroup comparison showed that citric acid had greater mean diameter $(1.75 \mathrm{~m})$ than EDTA $(1.24 \mathrm{~m})$. However, it appears that the mean diameter of the acidtreated groups is higher than the mean diameter of the control

\begin{tabular}{|c|c|c|c|c|c|c|}
\hline Variable & Material & Mean & SD & t-value & $p$-value & Significance \\
\hline Tubule diameter & $\begin{array}{l}\text { Citric acid } \\
\text { EDTA }\end{array}$ & $\begin{array}{l}1.7530 \\
1.241\end{array}$ & $\begin{array}{l}0.1265 \\
0.0890\end{array}$ & -10.4681 & $<0.01$ & $\mathrm{~S}$ \\
\hline
\end{tabular}

Table 5: Mean comparison of total surface area occupied by the dentinal tubule orifices between different treatment groups

\begin{tabular}{llllllc}
\hline Variable & Material & Mean & SD & t-value & p-value & Significance \\
\hline Surface area & Citric acid & 261.2067 & 53.3280 & 6.1962 & $<0.01$ & S \\
& EDTA & 126.3222 & 43.5304 & & & \\
\hline
\end{tabular}

NS: Nonsignificant; S: Significant 
specimens $(1.05 \mathrm{~m})$ in a study done by Labahn $\mathrm{R}$ et al. ${ }^{5}$ This widening of dentinal tubule orifices was due to the preferential demineralization of peritubular dentin. The results of this study were similar to the studies done by $\mathrm{H}$ anes PJ and Labahn R. ${ }^{4,6}$ However, this study is in contrast to studies done by Lafferty and Lasho where the dentinal tubules and the dentin between the tubules were affected to the same degree by either of the acid solutions. ${ }^{7,8}$

Intergroup comparison showed citric acid had greater mean total surface area occupied by the dentinal tubules than EDTA. This was due to the greater diameter of the dentinal tubules obtained in citric acid groups. This finding was in agreement with the study done by Hanes PJ et al who compared between citric acid and EDTA. B aker et al ${ }^{9}$ have clearly demonstrated the superiority of citric acid in comparison to EDTA when applied for 5 minutes on planed dentin root surfaces.

Ruggeri et al ${ }^{10}$ presented reliable findings using monoclonal antibodies and field emission in-lens scanning electron microscopy (FEISEM ) showing that both citric acid and EDTA treatments are able to etch and expose collagen fibrils and proteoglycans without any degradation of dentin collagen matrix.

Study done by $\mathrm{N}$ atalia et al ${ }^{11}$ to compare four of the most frequently used chemical root conditioners according to their efficiency on smear layer removal and dentin tubule widening showed that citric acid was the most effective followed by tetracycline- $\mathrm{HCl}$, phosphoric acid and EDTA. This information can be of value as an extraparameter for choosing one of them for root conditioning.

The results of this study are limited to the physical finding of root surface changes and do not present in vivo differences that may result from the physiologic effect of these acids. Differences between our results and those of other studies may be related to the disease status of the dentine specimens utilized, the demineralizing agent or a combination of these variables. A dditional studies of these variables with better standardization are needed for better understanding.

\section{CONCLUSION}

Since, Register and Burdick ${ }^{12}$ compared root conditioning with citric acid ( $\mathrm{pH} 1$ for 2-3 $\mathrm{min}$ ) and other chemical substances and found optimal cementogenesis and connective tissue new attachment, several investigators have devoted considerable time studying conditioning agents to improve periodontal regeneration. U nfortunately, numerous and often uncontrolled histological and clinical studies have created controversy and confusion about the positive or negative effects of those agents. ${ }^{13}$
B oth the acids are effective in removing the smear layer and exposing the collagen fibers. Citric acid is more effective in removing the smear layer and exposing the root collagen compared with EDTA. Citric acid causes greater degree of morphological alterations (mean diameter, mean total surface area occupied by dentinal tubules) than EDTA . Citric acid is considered to be a better root conditioning agent. H owever, the use of EDTA cannot be ruled out.

\section{CLINICAL SIGNIFICANCE}

Studies have suggested that the presence of smear layer interposed between the root surface and the adjacent connective tissue may serve as a physical barrier to the development of a connective tissue attachment to the root surface. Our present study, supports the hypothesis that demineralizing agents can be used as an adjunct to the periodontal treatment aiming at restoring the biocompatibility of roots and hel ps in choosing an appropriate agent for root conditioning.

\section{REFERENCES}

1. A leo JJ, De R enzis FA, Farber PA. In vitro attachment of human gingival fibroblasts to root surfaces. J Periodontol 1975;46: 639-45.

2. Bloml of J, Blomlf LB, Lindskog SF. Smear layer removal and collagen exposure after nonsurgical root planing followed by etching with an EDTA gel preparation. J Periodontol 1996;67:841-45.

3. Bloml of J, Bloml of L B, Lindskog SF. Smear layer formed by different root planing modalities and its removal by ethylenediaminetetra acetic acid gel preparation. Int Periodont Rest Dent 1997;17:243-49.

4. Henes PJ , O'Brien NJ, Garnick JJ. A morphologic comparison of radicular dentinefollowing root planing and treatment with citric acid or tetracycline HCL. J Clin Periodontol 1991;18:660-68.

5. Rosa wen C, Caffesse RG, M orrison EC, Nasjletti CE, Parikh UK. In vitro effects of citric acid application technique on dentine surfaces. J Periodontol 1992;63:883-89.

6. Labahn R, Fahrenbach WH, Clark SM, Lie T. Root dentine morphology after different modes of citric acid and tetracycline HCL conditioning. J Periodontol 1992; 303-09.

7. L afferty TA, Gher ME, Gray JL. Comparative SEM study on the effect of acid etching with tetracycline $\mathrm{HCL}$ or citric acid on instrumented periodontally involved root surfaces. J Periodontol 1993;64:689-93.

8. Lasho DJ, O'leary TJ, Kafrawy AH. A scanning electron microscope study of the effects of various agents on instrumented periodontally involved root surfaces. J Periodontol 1983;54: 210-20.

9. Baker DL, Stanley Pavlow SA, Wikesjö UME. Fibrin clot adhesion to dentin conditioned with protein constructs: $A n$ in vitro proof-of-principle study. J Clin Periodontol 2005;32:561-66.

10. Ruggeri A, Prati C, M azzoni A, Nucci C, Di L enarda R, M azzotti $G$, et al. Effects of citric acid and EDTA conditioning on exposed root dentin: A $n$ immunohistochemical analysis of collagen and proteoglycans. Arch Oral Biol 2007;52:1-8. 
11. Nathalia $G A, M$ aria $L R, F$ abiana $H, M$ arcus $G S, A$ driana $C P$, et al. Comparison among four commonly used demineralizing agents for root conditioning. A scanning electron microscopy. J Appl Oral Sci 2011;19(5):469-75.

12. Register AA, Burdick FA. A ccelerated reattachment with cementogenesis to dentin demineralized in situ. I. Optimun range. J Periodontol 1975;46:646-55.

13. Mariotti A. Efficacy of chemical root surface modifers in the treatment of periodontal disease. A systematic review. Ann Periodontol 2006;8:205-26.

\section{ABOUT THE AUTHORS}

\section{Sunkara Shree Ramalinga Prasad}

Professor, Department of Periodontics, Drs Sudha and Nageswara Rao Siddhartha Institute of D ental Sciences, K rishna, A ndhra Pradesh India

\section{Chitturi Radharani}

Professor, Department of Pedodontics and Preventive D entistry, Gitam Dental College, Vizag, A ndhra Pradesh, India

\section{Siddhartha Varma}

Senior Lecturer, Department of Periodontics, School of Dental Sciences, K IM SDV, K arad, M aharashtra, India

\section{SV Kiran Kumar}

Senior L ecturer, Department of Pedodontics and Preventive D entistry MA Rangoonwala College of Dental Sciences and Research Centre Pune, M aharashtra, India

\section{Soumya Sinha}

Senior L ecturer, Department of Pedodontics and Preventive D entistry MA Rangoonwala College of Dental Sciences and Research Centre Pune, M aharashtra, India

\section{Bijle Mohammed Nadeem Ahmed (Corresponding Author)}

Postgraduate Student, Department of Pedodontics and Preventive Dentistry, M A Rangoonwala College of Dental Sciences and Research Centre, Pune, Maharashtra, India, Phone: 9822884874 e-mail: info@drnadeembijle.com/nbijle@yahoo.co.in 